\title{
Size-dependent interface adhesive energy and interface strength of nanostructured systems
}

\author{
L.H. Liang ${ }^{\text {a, } *}$, H. Wei $^{\text {b }}$, X.N. Li ${ }^{\text {a }}$, Y.G. Wei ${ }^{\text {a }}$ \\ a LNM, Institute of Mechanics, Chinese Academy of Sciences, Beijing 100190, China \\ ${ }^{\mathrm{b}}$ Institute of Metal Research, Chinese Academy of Sciences, Shenyang 110016, China
}

\section{A R T I C L E I N F O}

\section{Article history:}

Received 9 April 2013

Accepted in revised form 22 October 2013

Available online 30 October 2013

\section{Keywords:}

Interface binding strength

Interface adhesive energy

Surface energy

Interface energy

Nanostructure

\begin{abstract}
A B S T R A C T
An analytical model about the size-dependent interface adhesive energy and the interface binding strength was developed based on the size-dependent surface energy and the interface cohesive zone model. The model predicts the enhanced interface binding strength between the nanostructured ceramic coatings and the bulk metallic substrates compared to that between the conventional ceramic coatings with micron-scale grains and the same substrates. The interface binding strength between the ceramic coatings and the metallic substrates was measured by the tensile adhesion test method. The result shows that the interface binding strength of the nanostructured coatings with grain diameter of average $70 \mathrm{~nm}$ increases by $86 \%$. The experimental result is in agreement with the theoretical prediction.
\end{abstract}

(c) 2013 Elsevier B.V. All rights reserved.

\section{Introduction}

Interface binding strength characterizes the stability of interfaces and reliability of the related devices such as microelectronic integrated circuits, Li-ion batteries and blades of gas turbine engines, etc. For example, ceramic coatings are usually used to provide thermal insulation for interior metallic components of the blades from the hot gas stream [1,2]. Once the interface between the ceramic coatings and metallic substrates fractured and the coat flaked off, the metallic components exposed in the high temperature environment would fail. The interface cohesive zone (ICZ) model is usually used to describe the mechanical behavior of the crack tip in the interface fracture process [3,4], which reflects the relation between the separating stress $\sigma$ and the displacement $\delta$ of the interfacial atoms. There are two important parameters in the ICZ model, one is the interface fracture strength (i.e. the interface binding strength) $\sigma_{f}$, the other is the interface fracture toughness (i.e. the interface adhesive energy) $\Gamma$. As Fig. 1 showed, $\Gamma=\int_{0}^{\delta_{f}} \sigma d \delta$, where $\delta_{f}$ is the interface fracture displacement. $\Gamma$ equals the area under the stressdisplacement curve (i.e. the cohesive curve) and reflects the needed work per unit area to separate the interface. The ICZ model is a macroscopic phenomenological model, the interface binding strength for the actual materials is usually the order of mega Pascal and the interface fracture displacement is the order of micron [3-5]. From point of view of atomic scale, the relation between the attraction force of the atoms in the interface cohesive zone and the atomic stretch distance is described by the interface cohesive potential $[4,6]$. The atomic scale simulation of the interface tension between metals and ceramics shows the

\footnotetext{
* Corresponding author.

E-mail address: lianglh@Inm.imech.ac.cn (L.H. Liang).
}

similar curve as shown in Fig. 1 [6], where $\sigma_{f}$ is the order of giga Pascal and $\delta_{f}$ is the order of angstrom. This trans-scale similarity from microscopic to macroscopic scales reflects the size effect of the related interface mechanical properties.

Recently, the studies have indicated that interface energy $\gamma_{i}$, the excess free energy per unit area of the system due to the existence of the interface, shows the size-dependence for the nanoscaled heterogeneous thin films, $\gamma_{i}$ decreases with reducing thickness of thin films [7-9]. The reduced interface energy characterizes the enhanced stability of the interface [9]. Does the interface between the nanostructured ceramic coatings and the bulk alloy substrates possess reduced interface energy and enhanced interface binding strength compared to that between the conventional ceramic coatings and the same substrates? Understanding this point is significant in guiding the application of nanomaterials not only in the engineering of thermal barrier coatings, but also in microelectronic field. The study has showed the scale effect of friction and adhesion of MEMS/NEMS [10]. From point of view of solid state physics, the interface adhesive (cohesive) energy is the absolute value of the minimum of the potential energy function of the interface between different (same) materials, the interface stress is the differential of the interface potential, and the interface strength is the maximum of the interface stress function [6,9]. However, the interface potential itself is usually unknown and difficult to be obtained for the actual heterogeneous materials not like the cohesive potential function (universal binding relation) for homogeneous materials such as metals, ceramics and compounds.

In this paper, an analytic thermodynamic model about the size effect of the interface adhesive energy between the ceramic coatings and the metallic substrates was developed. The change of the interface strength between the nanostructured $\mathrm{ZrO}_{2}$ ceramic coatings with grain diameter 


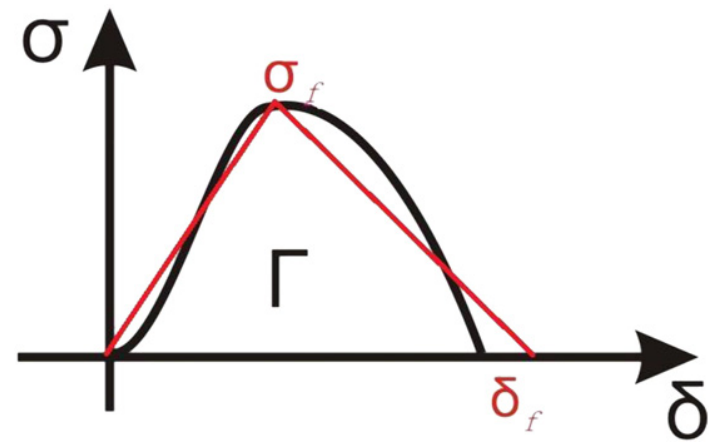

Fig. 1. The schematic illustration of the interface cohesive zone model describing the relation between the separating stress $\sigma$ and the displacement $\delta$ of the interfacial atoms. The triangular curve (bilinear functions) is the simplified form of the cohesive relation. The interface adhesive energy $\Gamma$ and the interface binding strength $\sigma_{f}$ are two important parameters.

of average $70 \mathrm{~nm}$ and the metallic substrates, compared to that between the conventional ceramic coatings with the bulk splat grains and the same substrates, was checked.

\section{Theoretical model}

2.1. Triangle simplification of ICZ model and assumption of interface fracture displacement

According to the cohesive curve of the ICZ model, the triangle form of the stress-displacement curve (i.e. the bilinear functions) was considered simply as the first order approximation as shown in Fig. 1, since the shape of the curve is not intrinsic for the interface properties compared to two parameters of the strength and the toughness [3-6,11], i.e.,

$\Gamma=\sigma_{f} \delta_{f} / 2$.

According to Eq. (1), if there is no large change in the interface adhesive energy $\Gamma$ or $\Gamma$ increases, the interface strength $\sigma_{f}$ will increase as the interface fracture displacement $\delta_{f}$ decreases.

Since two new surfaces of the ceramic coating and the metallic substrate will form after fracturing of their interface, the change of the interface fracture displacement may be obtained by the change of the surface roughness. The surface roughness of the nanostructured ceramic coatings (nano-coatings) has been found to be about 2.6-3 times lower than that of the conventional ceramic coatings, which is about several microns $[12,13]$ as shown in Table 1 . Since the metallic substrates (including the alloy bond coats and the metallic matrixes here) are same both for the nano-coating and the conventional ceramic coating systems, the ratio of the interface fracture displacement of the nano-

Table 1

The surface roughness of the nano-ceramic coatings is smaller than that of the conventional ones.

\begin{tabular}{llccc}
\hline Samples & \multicolumn{4}{l}{ Surface roughness $(\mu \mathrm{m})$} \\
\hline Nano $(n)$ & $3.5[13]$ & $2.75[13]$ & $4.5 \pm 0.9[12]$ & $0.80 \pm 0.14^{\mathrm{a}}$ \\
Conventional $(b)$ & $9.8[13]$ & $9.09[13]$ & & $2.15 \pm 0.50^{\mathrm{a}}$ \\
\hline
\end{tabular}

a Our test results obtained by use of the Synthesis Measuring Profilometer AF-LI04 based on the contact measuring method. Before the test, the surface of the coatings was abraded, polished, and cleaned by the ultrasonic radiation for $30 \mathrm{~min}$. In the test, the numbers of measurement lines along two orthogonal directions were both chosen to be 3 , the tracing length is $2.5 \mathrm{~mm}$, and the data were recorded at the interval of $1 \mu \mathrm{m} .10$ specimens were measured and the average results were taken. The test method can also be referred to Ref. [12]. coating systems to that of the conventional ones was assumed to be same as the ratio of the surface roughness of the nano-coatings to that of the conventional ceramic coatings. So $\delta_{f}$ of the nano-coating systems is also 2.6-3 times lower than that of the conventional ones, i.e., $\delta_{f b} \in(2.6-3) \delta_{f n}$, where the subscripts $b$ and $n$ represent the conventional coatings with bulk grains and the nano-coatings with nanoscaled grains, respectively. The assumption of the reduced interface fracture displacement of the nano-coating systems is reasonable considering the reduced scale of the microstructure of the ceramic coatings, which will also be validated in the Results and discussion.

\subsection{Thermodynamics of size-dependent interface adhesive energy}

According to the definition of the thermodynamics, the interface adhesive energy $\Gamma$ is related to the surface energies of the ceramic coatings and the metallic substrates after separation of the interface between both, and the interface energy $\gamma_{i}$ of the interface between the ceramics and the metallic alloys before separating,

$\Gamma=\gamma_{m}+\gamma_{c}-\gamma_{i}$

where $\gamma_{m}$ and $\gamma_{c}$ are the surface energy of the metallic substrates and the ceramic coatings, respectively. The surface energy of the metallic substrates (Ni alloy bond coats and the steel matrixes as the whole metallic substrates) was taken as the surface energy of Ni approximately $\gamma_{m}=2.42 \mathrm{Jm}^{-2}$ [14], which is the same for the nano-coating systems and the conventional ceramic coating systems. For the nano-coating systems, on one hand, the surface energy of the ceramic coatings $\gamma_{c}$ may decrease due to the reduced scale of the microstructure, on the other hand, the interface energy $\gamma_{i}$ of the ceramic/metal interfaces also decreases despite of minuteness. The rate of the decrease of the surface energy is generally larger than that of the interface energy for homogeneous materials [8]. If this is also the case here, $\Gamma$ will decrease for the nano-systems.

The surface energy is the excess free energy per unit area of the system due to the existence of the surface and the imperfection of the coordinate number of atoms on the surface. The surface energy $\gamma_{c b}$ of the bulk $\mathrm{ZrO}_{2}$ ceramics can be obtained based on the rule of the surface breaking bond [15], $\gamma_{c b}=\left[2-Z_{s} / Z_{b}-\left(Z_{s} / Z_{b}\right)^{1 / 2}\right] E_{b} / 2$, where $Z_{s}$ and $Z_{b}$ denote the coordinate number of atoms on the surface and in the bulk, respectively, and $E_{b}$ is the cohesive energy of the bulk crystals [15]. According to the above equation, the surface energy of the conventional bulk $\mathrm{ZrO}_{2}$ coatings is $\gamma_{c b}=15.6 \mathrm{Jm}^{-2}$ considering the simple cube structure of $\mathrm{ZrO}_{2}$ crystals with $Z_{s}=8$ at (100) face and $Z_{b}=12$ as the average and $E_{\mathrm{b}}=119 \mathrm{eV}$ per $\mathrm{ZrO}_{2}$ molecular [16]. The above equation also shows that the surface energy is proportional to the cohesive energy. Therefore, the size effect of the surface energy can be obtained by the size effect of the cohesive energy [17], $\gamma_{c n} / \gamma_{c b}=E_{n} / E_{b}=[1-1 /(D /$ $\left.\left.D_{c}-1\right)\right] \exp \left\{-2 S_{c} /\left[3 R\left(D / D_{c}-1\right)\right]\right\}$, where $\gamma_{c n}$ is the surface energy of the nano-ceramics, $E_{n}$ is the cohesive energy of the nano-ceramics, $D$ is the thickness of single-crystal thin films or the diameter of nanoparticles, and was taken as the grain diameter of the polycrystalline ceramic coatings here, $D_{c}=h / 2$ is the minimum critical size corresponding to the solid-vapor transition with the average bond length $h$ of the crystals, $S_{c}$ is the sublimation entropy of the crystals, and $R$ is the ideal gas constant [17]. Considering that $h=0.324 \mathrm{~nm}$ for $\mathrm{ZrO}_{2}$ [16], and the grain diameter $D$ is about $40-100 \mathrm{~nm}$ for the nano-coatings based on the present preparation technology, the size-dependent surface energy can be simplified as

$\gamma_{c n}=\gamma_{c b} \exp \left[-S_{c} h /(3 R D)\right]$

where $S_{c}=E_{b} / T_{b}$ [16] with the boiling point $T_{b}=5273 \mathrm{~K}$ [18]. According to Eq. (3), considering the average grain size of $D=70 \mathrm{~nm}$, the surface energy of the nano-coatings decreases about 33\% compared to that of the conventional ceramic coatings due to the large sublimation 
entropy of $60 \mathrm{Jmol}^{-1} \mathrm{~K}^{-1}$, i.e., $\gamma_{c n}=0.67 \gamma_{c b}$. Note that the effect of thickness of the coatings was neglected since the thickness is the order of hundred microns both for the nano-coatings and the conventional ones.

The heterogeneous interface energy $\gamma_{i}$ between the ceramic coatings and the metallic substrates was taken as the average value of the homogeneous interface energies of the ceramics and the metallic alloys. The homogeneous bulk solid-solid interface energy $\gamma_{h b}$ can be calculated by the related thermodynamic parameters of the crystals [7-9], $\gamma_{h b}=4 h S_{v} H /(3 V R)$, where $S_{v}$ is the vibration part of the melting entropy, $H$ is the melting enthalpy, and $V$ is the molar volume of the crystals [7-9]. For the interface between the conventional ceramic coatings and the metallic substrates, the bulk interface energy $\gamma_{i b}$ is expressed as

$\gamma_{i b}=[2 /(3 R)]\left[h_{1} S_{v 1} H_{1} / V_{1}+h_{2} S_{v 2} H_{2} / V_{2}\right]$

where the subscripts 1 and 2 represent the metals and ceramics, respectively. For the nano-coating systems, there is no change in the homogeneous interface energy of the metallic alloys, and the homogeneous interface energy of the ceramic coatings decreases due to the nanostructurization. The size-dependent homogeneous solid-solid interface energy $\gamma_{h n}=\gamma_{h b}\left[1-D_{0} /(4 D)\right]$, where $D_{0}=3 h$ is the critical size of the particles corresponding to the solid-liquid transition [7-9]. Considering that $D_{0}=0.972 \mathrm{~nm}$, and the average diameter $D=70 \mathrm{~nm}$ for the $\mathrm{ZrO}_{2}$ grains, the size effect can be neglected. Therefore, the interface energy $\gamma_{i n}$ between the nano-coatings and the same metallic substrates is approximately equal to $\gamma_{i b}$. Note that the structure strain energy between the coatings and the substrates was also neglected considering the thickness of hundred microns of the coatings [7,9]. According to Eq. (4) and the related parameters $[2,19-22]$ in Table $2, \gamma_{i}=2.93 \mathrm{Jm}^{-2}$, the value of several joules per meter square is in agreement with the interface energy of a series of the interfaces between the metals and the ceramics [9].

\subsection{Prediction of interface binding strength}

According to Eq. (2), for the conventional ceramic coating systems, the interface adhesive energy is $\Gamma_{b}=\gamma_{m}+\gamma_{c b}-\gamma_{i}=15.1 \mathrm{Jm}^{-2}$, this is in agreement with the previous report of $5-20 \mathrm{Jm}^{-2}$ [1]. While for the nano-coating systems, the interface adhesive energy is $\Gamma_{n}=\gamma_{m}+\gamma_{c n}-\gamma_{i}=9.9 \mathrm{Jm}^{-2}$, which is about 1.5 times lower than that of the conventional ones due to the reduced surface energy of the nano-coatings, i.e., $\Gamma_{b}=1.5 \Gamma_{n}$. The result is consistent with the previous report that the interface adhesive energy may decrease with reducing size [11]. According to Eq. (1), combining $\Gamma_{b}=1.5 \Gamma_{n}$ with $\delta_{f b} \in(2.6-3) \delta_{f n}, \sigma_{f n} \in(1.7-2) \sigma_{f b}$ can be obtained, i.e., the interface binding strength for the nanostructured ceramic coating systems can increase 1.7-2 times even if $\Gamma$ decreases.

\section{Experimental}

\subsection{Sample preparation}

The samples with $\mathrm{ZrO}_{2}-8 \% \mathrm{Y}_{2} \mathrm{O}_{3}$ (YSZ) top coatings of about $140 \mu \mathrm{m}$ thickness (the thickness of the coatings for some samples is thicker or

Table 2

The related parameters in Eq. (4). For $\mathrm{ZrO}_{2}, h$ is calculated by the lattice volume [16], $V$ is calculated by the mass and the density [2], $S_{v}=S_{m}-R$ with the melting entropy $S_{m}=H / T_{m}$ [19], $T_{m}$ is the melting point. For metals, $S_{v}=S_{m}$.

\begin{tabular}{llllll}
\hline Materials & $h(\mathrm{~nm})$ & $S_{v}\left(\mathrm{Jmol}^{-1} \mathrm{~K}^{-1}\right)$ & $H\left(10^{3} \mathrm{Jmol}^{-1}\right)$ & $T_{m}(\mathrm{~K})$ & $V\left(\mathrm{~cm}^{3} \mathrm{~mol}^{-1}\right)$ \\
\hline $\mathrm{ZrO}_{2}$ & 0.3240 & 21.12 & $87.5[20]$ & $2973[2]$ & 20.54 \\
$\mathrm{Ni}$ & 0.2754 & 10.12 & $17.47[22]$ & 1726 & $6.59[22]$ \\
& {$[21]$} & & & {$[22]$} & \\
\hline
\end{tabular}

thinner), bonded on the medium carbon steel matrixes, were fabricated by the standard air plasma spray method [23]. The NiCrAlY interface bond coats of about $60 \mu \mathrm{m}$ thickness, between the ceramic top coatings and the metallic matrixes, were obtained using the high velocity oxygen fuel process [24]. Two kinds of ceramic coatings were prepared by the similar process $\left(\mathrm{Ar} / \mathrm{H}_{2}\right)$ on the Metco $7 \mathrm{M}$ atmospheric plasma spray equipment, and the METCO 9MP-DUAL type feedstock system was used. The detailed process parameters are shown in Table 3. The microstructure of one kind of ceramic coatings is in nanoscale prepared from the nanostructured YSZ powder (CM60, Institute of Process Engineering, Chinese Academy of Sciences), and the other is in micron-scale prepared from the conventional YSZ powder with the larger granularity (Metco 204CNS, Sulzer Metco, Westbury, NY, USA) by means of the standard parameters suggested by the manufacturer of the powder and torch (Sulzer Metco). The grain diameter of the nano-coatings is about $40-100 \mathrm{~nm}$ [24] (the microstructure detail can be referred to the TEM micrograph in Fig. 2(c) in Ref. [24]), agreeing with the theoretical estimation. For the bulk splat grains of the conventional ceramic coatings, the diameter is the order of hundred microns and the thickness is about several microns [2]. The total thickness of the samples is about $6 \mathrm{~mm}$, and the diameter is $25 \mathrm{~mm}$. The microstructure and the cross-section structure of the samples were observed by the FEI Tecnai G2 F20 transmission electron microscope (TEM) and the S-570 scanning electron microscope (SEM), respectively.

\subsection{Test of interface binding strength}

The interface binding strength between the ceramic coatings and the metallic substrates was measured by the standard tensile adhesion test $[25,26]$. The sample and the connection component were bonded by E-7 glue, which was roasted $3 \mathrm{~h}$ to be dry and solidified in the electrothermal constant temperature oven at $373 \mathrm{~K}$. The tension measurement was carried out on the AG-100KNG tension test machine, the tension rate is $1 \mathrm{~mm} / \mathrm{min}$. The schematic illustration of the measurement and the samples is shown in Fig. 2. The load per unit area at the interface fracture was taken as the interface binding strength. The fracture part was observed to confirm that the fracture occurred at the interface between the ceramic coatings and the metallic substrates (in detail between the ceramic coatings and the metallic alloy bond coats as observed in Fig. 6 of Ref. [26], the bond coats and the matrixes were considered as the whole metallic substrates here), and the data corresponding to the fracture occurring in the glue or in the ceramic coatings were deleted, since the strength of the glue or the ceramic coatings but not the interface strength is reflected in those cases.

\section{Results and discussions}

\subsection{Measurement results}

Fig. 3 shows the cross-section structure of the as-sprayed samples. The interface bond coat between the conventional ceramic coating and the metallic matrix can be seen clearly as shown in Fig. $3 \mathrm{~A}$, while the interface bond coat between the nano-ceramic coating and the metallic matrix becomes not obvious as shown in Fig. 3B, which may imply the better binding between the nano-coatings and the substrates. The measured average interface fracture strength $\sigma_{f n}$ between the nanocoatings and the substrates of 10 effective samples is about $52 \mathrm{MPa}$, the original data and the average error are shown in Table 4. While

Table 3

Processing parameters of the plasma spraying of the YSZ coatings.

\begin{tabular}{llllll}
\hline Power $(\mathrm{KW})$ & $d(\mathrm{~mm})$ & $\operatorname{Ar}\left(\mathrm{m}^{3} / \mathrm{h}\right)$ & $\mathrm{H}_{2}\left(\mathrm{~m}^{3} / \mathrm{h}\right)$ & $F(\mathrm{~g} / \mathrm{min})$ & $v(\mathrm{~mm} / \mathrm{s})$ \\
\hline 50 & $95-100$ & 2.4 & 0.3 & 40 & 500 \\
\hline Note: $d$ - spray distance, $F-$ feed rate, and $v$ - transverse speed of plasma gun.
\end{tabular}




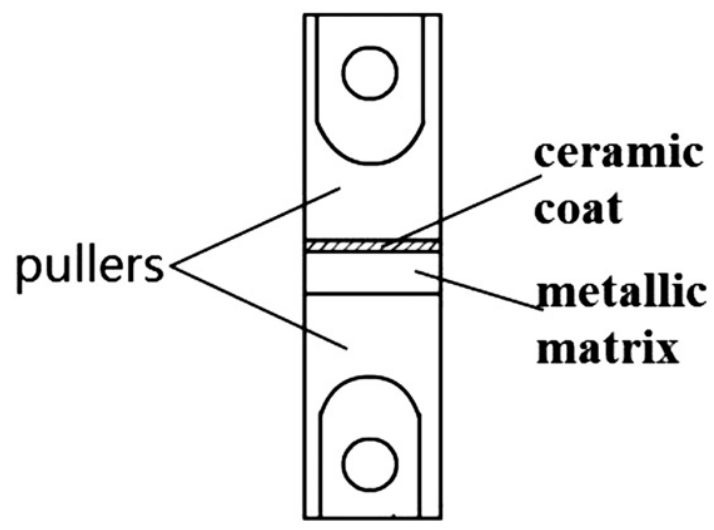

Fig. 2. The schematic illustration of the measurement of the interface binding strength between the ceramic coatings and the metallic substrates.

the average interface strength $\sigma_{f b}$ between the conventional coatings and the same substrates is $28 \mathrm{MPa}$, which is in agreement with the previous report of about $29 \mathrm{MPa}[26,27]$. Obviously, the interface strength of the nano-coating samples increases compared to that of the conventional ones, $\sigma_{f n}=1.86 \sigma_{f b}$, which is in agreement with the theoretical prediction (1.7-2) and validates the assumption of the interface fracture

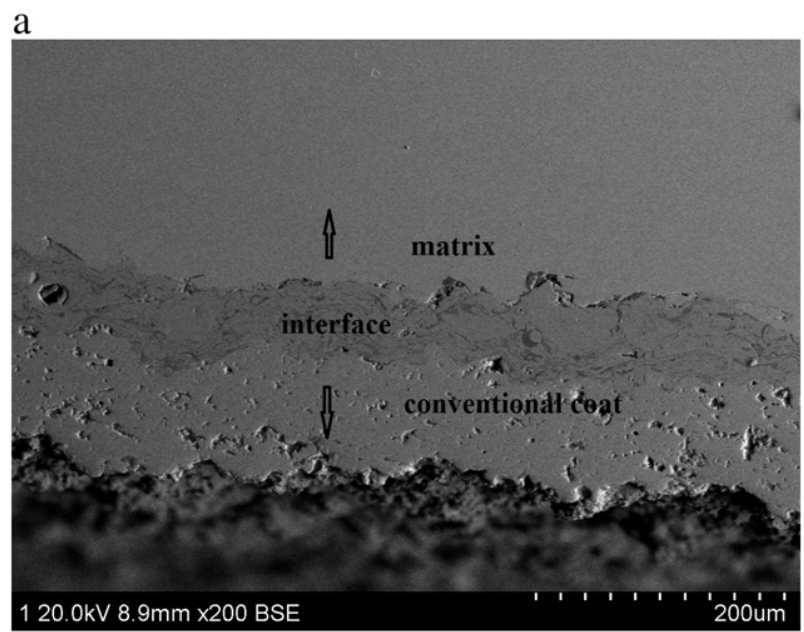

b

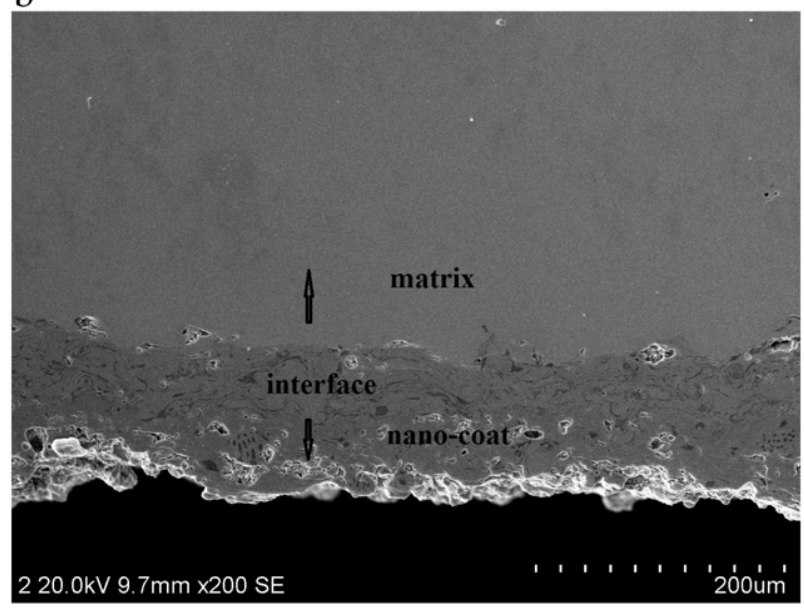

Fig. 3. SEM micrograph of the cross-section of the initial as-sprayed samples with the conventional coating (a), and the nano-coating (b), respectively. The interface between the conventional coating and the substrate is clearer.
Table 4

The original data of the interface strength test. The tests were carried out 2 times, at least 5 effective samples per time. The error in the 1st test of the nano-systems is larger, but even the smallest strength is larger than that of the conventional ones.

\begin{tabular}{|c|c|c|c|c|c|c|}
\hline Samples & Measured streng & h (MPa) & & & & \\
\hline $\begin{array}{l}\text { Nano }(n) \\
\text { Conventional }(b)\end{array}$ & $\begin{array}{l}35.10 \quad 31.16^{a} \\
28 \pm 5^{c}\end{array}$ & 39.14 & 43.46 & 50.55 & 42.44 & $62 \pm 5^{b}$ \\
\hline
\end{tabular}

${ }^{\mathrm{a}}$ Fracture in glue in the 1 st test.

b The average results of 5 effective samples in the 2 nd test.

c The average results of 10 effective samples in two tests.

displacement in turn. The result is reasonable since the reduced scale of the microstructure of the coatings decreases the amplitude and wavelength of the undulation of the interface, the interface separation displacement decreases, and the interface adhesive energy reflects the intrinsic property of the interface despite of the light change, thus the interface strength enhances. Certainly, the detailed preparation parameters and conditions will affect the interface strength greatly, only the pure size effect is discussed in this work, which can be as a reference at the same other conditions. For example, the different surface roughness of the bond coats will induce different adhesive properties between the ceramic top coatings and the alloy bond coats, but as the bond coats are same, the different scales of the microstructure of the ceramic coatings will induce different adhesive properties between the ceramics and the alloys.

\subsection{Theoretical analysis and discussion}

The above calculated and measured interface cohesive parameters $\Gamma$ and $\sigma_{f}$ between the ceramic coatings and the alloy substrates were summarized in Table 5 . The interface fracture displacement $\delta_{f}$ can be obtained based on the Eq. (1) when $\Gamma$ and $\sigma_{f}$ are known, but the critical displacement, at which the interface strength $\sigma_{f}$ is reached, is unknown. As previously mentioned, the shape of the cohesive curve is not intrinsic, which can be simplified as the triangle (bilinear functions), the trapezoid, and the exponential form, etc [3-6,11]. According to the virtual internal bond model as an exponential form of the cohesive curve [28], the interface separating stress is expressed as

$\sigma=k \delta \exp \left(-\delta / \delta_{c}\right)$

where $k$ is the slope of the initial stage of the stress-displacement curve characterizing the interface modulus, and $\delta_{c}$ is the critical displacement corresponding to the interface strength $\sigma_{f}$. Eq. (5) is in agreement with the universal binding relation among atoms of metals [29], and the stress function is the differential form of the binding energy function. But the virtual internal bond model describes the cohesive relation among particles of materials [28], not among atoms. When $\delta=\delta_{c}$,

$\sigma=\sigma_{f}=k \delta_{c} e^{-1}$

based on Eq. (5), and the interface toughness

$\Gamma=k \delta_{c}^{2}$

as the integral of the stress function. Combining Eqs. (6) with (7), the

Table 5

Comparison of the interface cohesive parameters between the nano-coatings/substrates and the conventional coatings/substrates.

\begin{tabular}{lcllll}
\hline Samples & $\Gamma\left(\mathrm{Jm}^{-2}\right)$ & $\sigma_{f}(\mathrm{MPa})$ & $\delta_{f}(\mu \mathrm{m})$ & $k\left(10^{12} \mathrm{Nm}^{-3}\right)$ & $\delta_{c}(\mu \mathrm{m})$ \\
\hline Nano $(n)$ & 9.9 & 52 & 0.38 & 2020 & 0.07 \\
Conventional $(b)$ & 15.1 & 28 & 1.10 & 378 & 0.20 \\
\hline
\end{tabular}




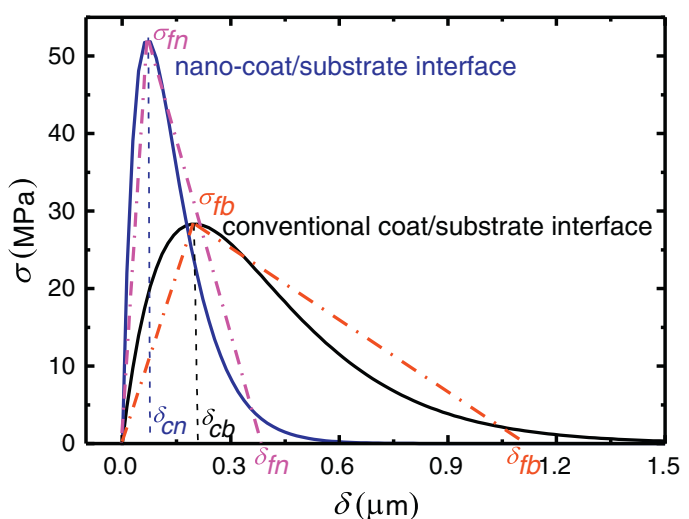

Fig. 4. The higher interface strength $\sigma_{f}$ and the smaller critical (fracture) displacement $\delta_{c}$ $\left(\delta_{f}\right)$ of the nano-coatings/substrates compared to the interface parameters of the conventional coatings/substrates. The solid curves and the dash dot curves are based on the exponential function and the bilinear functions, respectively, referring to the parameters in Table 5.

parameters $k$ and $\delta_{c}$ for the interfaces of the nano-coatings/substrates and the conventional coatings/substrates, respectively, can be calculated based on $\Gamma$ and $\sigma_{f}$ in Table 5. By using all of the parameters in Table 5, the cohesive curves, not only the exponential form, but also the bilinear form, can be obtained as shown in Fig. 4. Note that the interface fracture displacement in the exponential form tends to be infinite based on the similar interaction potential to that of atomic binding, the approximate $\delta_{f}$ was cut off.

The results in Fig. 4 (or Table 5) show that $\delta_{c b} / \delta_{c n}=\delta_{f b} / \delta_{f n}=2.8$, which is in agreement with the assumed value of $\delta_{f b} / \delta_{f n}$ in the theoretical model (2.6-3). Furthermore, $\delta_{c b} / \delta_{f b}=\delta_{c n} / \delta_{f n}$ can be obtained, meaning that the ratio of the critical displacement to the fracture displacement is a constant for the interfaces between the ceramics and the substrates with the same materials, but not dependent on the size or the thickness of the interfacial materials, which agrees with the previous experimental observation [30], and validates the theoretical assumption in turn. The interface fracture displacement is about 5-6 times of the critical displacement, agreeing with the previous discussion, too [3].

It can also be seen from Fig. 4 (Table 5) that the critical displacement $\delta_{c n}$, corresponding to the interface strength $\sigma_{f n}$, is about $70 \mathrm{~nm}$, which is just the average grain size of the nano-coatings. The result is reasonable because the interface fracture strength corresponds to the needed stress to break the interfacial basic unit (minimum material particles), i.e., the grains of the nano-ceramic coatings for nano-systems since the microstructure of the interface bond coats is larger. Therefore, the physical meaning of the critical displacement is the characteristic size of the material particles as the minimum microstructure unit at the interface. While $\delta_{c b}$ is about $200 \mathrm{~nm}$, which corresponds to the size of the single phase in the NiCrAlY interface bond coats [13] as shown in Fig. 5 (the TEM micrograph of the interface bond coats), the single phase is as the minimum unit at the interface for the conventional coating systems, since the grains of the conventional ceramic coatings are much larger. As previous discussed, the interface fracture occurs between the ceramic top coatings and the bond coats in detail usually [26].

\section{Conclusions}

In summary, the size-dependent interface adhesive energy model was developed based on the size-dependent surface energy. And the enhanced interface strength between the nanostructured coatings and the metallic substrates, compared to that between the conventional coatings and the same substrates, was predicted based on the interface cohesive zone model. The experimental result is in agreement with the theoretical prediction. The trans-scale mechanism of the interface mechanical behavior of the nanostructured systems is resulted from the

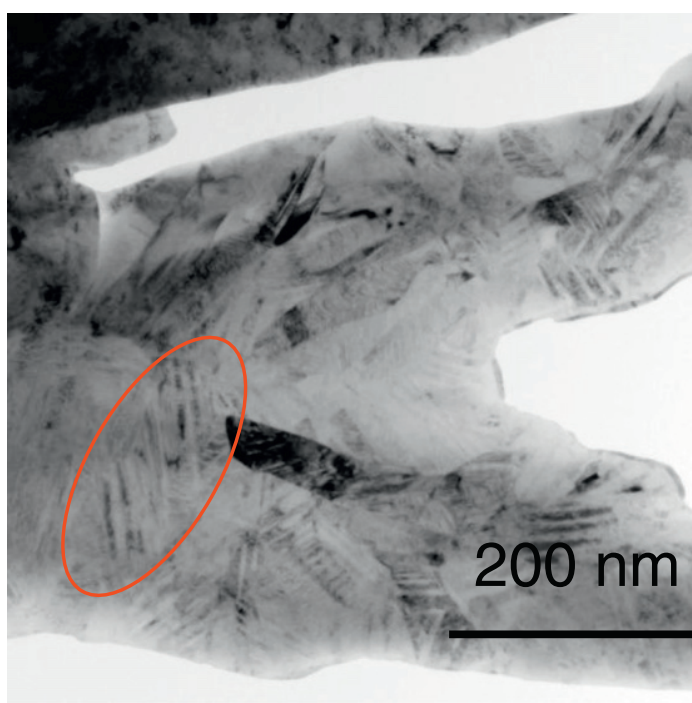

Fig. 5. TEM micrograph of the NiCrAlY interface bond coats (scale bar $200 \mathrm{~nm}$ ), the size of the single phase (encircled in the loop) is about $200 \mathrm{~nm}$.

size effect of the interface cohesive relation. Although the interface adhesive energy decreases slightly due to the decrease of the surface energy of the nano-coatings, the interface strength increases due to the obvious decrease of the interface separate displacement related to the decreased scale of the microstructure.

\section{Acknowledgments}

This work was supported by the National Basic Research Programs of China (Nos. 2012CB937500 and 2010CB631206), the NSFC grants of China (Nos. 10802088, 11372318, 11023001, 11021262, 10932011, 91216108 and 51071164), the CAS/SAFEA International Partnership Program for Creative Research Teams, and the opening fund of the LNM.

\section{References}

[1] A.G. Evans, D.R. Mumm, J.W. Hutchinson, G.H. Meier, F.S. Pettit, Prog. Mater. Sci. 46 (2001) 505

[2] N.P. Padture, M. Gell, E.H. Jordan, Science 296 (2002) 280.

[3] A. Needleman, J. Appl. Mech. Trans. ASME 54 (1987) 525:

V. Tvergaard, J.W. Hutchinson, J. Mech. Phys. Solids 40 (1992) 1377;

M. Lane, R.H. Dauskardt, A. Vainchtein, H.J. Gao, J. Mater. Res. 15 (2000) 2758.

[4] O. Nguyen, M. Ortiz, J. Mech. Phys. Solids 50 (2002) 1727;

A. Siddiq, S. Schmauder, Y.G. Huang, Int. J. Plast. 23 (2007) 665

[5] H.F. Zhao, Y.G. Wei, Int. J. Fract. 144 (2007) 103.

[6] Y. Long, N.X. Chen, W.Q. Zhang, J. Phys. Condens. Matter 17 (2005) 2045; Y.G. Wei, J.W. Hutchinson, Philos. Mag. 88 (2008) 3841; Y. Jiang, J.R. Smith, A.G. Evans, Appl. Phys. Lett. 92 (2008) 141918.

[7] G. Ouyang, L.H. Liang, C.X. Wang, G.W. Yang, Appl. Phys. Lett. 88 (2006) 091914.

[8] Q. Jiang, H.M. Lu, Surf. Sci. Rep. 63 (2008) 427.

[9] L.H. Liang, X.M. You, H.S. Ma, Y.G. Wei, J. Appl. Phys. 108 (2010) 084317.

[10] B. Bhushan, N.S. Tambe, Nanotechnology 15 (2004) 1561.

[11] Y.G. Wei, J.W. Hutchinson, J. Mech. Phys. Solids 45 (1997) 1137.

[12] R.S. Lima, A. Kucuk, C.C. Berndt, Surf. Coat. Technol. 135 (2001) 166

[13] Y.J. Zhang, Research of Oxidation Behaviors of NiCrAlY Coatings and Microstructure of YSZ NanoTBCs on a Single Crystal Superalloy, (PH. D thesis) Institute of Metal Research, Chinese Academy of Sciences, 2003. 97.

[14] L. Vitos, A.V. Ruban, H.L. Skriver, J. Kollár, Surf. Sci. 411 (1998) 186.

[15] Q. Jiang, H.M. Lu, M. Zhao, J. Phys. Condens. Matter 16 (2004) 521.

[16] A.L. Ortiz, J. Sánchez-González, L.M. González-Méndez, F.L. Cumbrera, Ceram. Int. 33 (2007) 705.

[17] Q. Jiang, J.C. Li, B.Q. Chi, Chem. Phys. Lett. 366 (2002) 551.

[18] http://zh.wikipedia.org/zh-cn/ZrO2.

[19] Z. Zhang, M. Zhao, Q. Jiang, Semicond. Sci. Technol. 16 (2001) L33.

[20] D.C. Jia, in: Y. Zhou (Ed.), Ceramic Materials, 2nd ed., Science Publisher, Beijing, 2004, p. 320.

[21] H.W. King, in: R.W. Cahn (Ed.), Physical Metallurgy, North-Holland Pub. Co., Amsterdam, 1983, p. 64. 
[22] Sargent-Welch Scientific Company, Periodic Table of the Elements, Skokie, Illinois, 1980. 1.

[23] Z. Chen, R.W. Trice, M. Besser, X. Yang, D. Sordelet, J. Mater. Sci. 39 (2004) 4171.

[24] L.H. Liang, H. Wei, X.C. Chang, W. Xu, X.N. Li, Y.G. Wei, Int. J. Heat Mass Transf. 65 (2013) 219.

[25] ASTM, Standard test method for adhesion or cohesion strength of thermal spray coatings, ASTM Standard C 633-01, West Conshocken, PA, USA2001.
[26] C.R.C. Lima, J.M. Guilemany, Surf. Coat. Technol. 201 (2007) 4694

[27] P.F. Zhao, C.A. Sun, X.Y. Zhu, F.L. Shang, C.J. Li, Surf. Coat. Technol. 204 (2010) 4066

[28] H.J. Gao, P. Klein, J. Mech. Phys. Solids 46 (1998) 187; P. Klein, H.J. Gao, Eng. Fract. Mech. 61 (1998) 21.

[29] J.H. Rose, J. Ferrante, J.R. Smith, Phys. Rev. Lett. 47 (1981) 675

[30] G.F. Ji, Z.Y. Ouyang, G.Q. Li, S. Ibekwe, S.S. Pang, Int. J. Solids Struct. 47 (2010) 2445. 